\title{
ARCS IN INVERSE LIMITS ON [0, 1$]$ WITH ONLY ONE BONDING MAP
}

\author{
WILLIAM S. MAHAVIER
}

1. Introduction. In this note, by a continuum we mean a nondegenerate, compact, connected metric space. It is known ([2] or [5]) that a continuum is chainable if and only if it is homeomorphic to the inverse limit of a sequence of maps from $[0,1]$ onto $[0,1]$. Indeed, the mappings may be required to be piecewise linear. Henderson has shown that a pseudo arc can be obtained as an inverse limit on $[0,1]$ with only one bonding map [3], and the author has shown that not every chainable continuum can be so obtained [4]. We now show that if $M$ is an inverse limit on $[0,1]$ with only one bonding map, and the bonding map is piecewise monotone, then every subcontinuum of $M$ contains an arc. Thus the set of all such continua is a proper subset of the set of chainable continua which are inverse limits on $[0,1]$ with only one bonding map.

2. Definitions and notation. If each term of the sequence $g=\left\{g_{i}\right\}$ maps $[0,1]$ onto $[0,1]$, then the inverse limit of $g$, denoted by $\lim g$, is the subspace of the infinite cartesian product $[0,1]^{\infty}$ consisting of all number sequences $\left\{x_{i}\right\}$ such that for each $i>0, g_{i}\left(x_{i+1}\right)=x_{i}$. If $f$ maps $[0,1]$ onto $[0,1]$, then $\lim f \operatorname{denotes} \lim g$ where $g=f, f, \cdots$. By an interval we mean a nondegenerate closed subinterval of $[0,1]$, and the statement that $A$ is an inverse sequence (for $f$ ) means that $A$ is a sequence $A_{1}, A_{2}, \ldots$ such that if $i>0, A_{i}$ is degenerate or an interval and $f\left(A_{i+1}\right)=A_{i}$. The set of all points of $\lim f$ such that for each $i>0, x_{i} \in A_{i}$ is denoted by $\lim (f, A)$. By a subinverse sequence of $A$ is meant an inverse sequence $B=\left\{B_{i}\right\}$ such that if $i>0, B_{i} \subseteq A_{i}$. If each of $I$ and $I^{\prime}$ is an interval, the statement that $f$ maps $I$ onto $I^{\prime}$ efficiently means that $f(I)=I^{\prime}$ and no interior point of $I$ maps onto an endpoint of $I^{\prime}$.

3. Arcs in chainable continua. Our object in this section is to prove the following:

THEOREM. If $f$ is a piecewise monotone function from $[0,1]$ onto $[0,1]$ then each subcontinuum of $\lim f$ contains an arc.

Proof. Suppose there is a subcontinuum of $\lim f$ which contains no arc. Then there is an inverse sequence $A=\left\{A_{i}\right\}$ such that $\lim (f, A)$ contains no arc. There is an increasing number sequence $x_{0}=0, x_{1}$,

Received by the editors June 3, 1968. 
$\cdots, x_{n}=1$ such that if $0<i \leqq n, f \mid\left[x_{i-1}, x_{i}\right]$ is monotone. Let $M$ denote the set of images under $f$ of the numbers in this sequence and let $\epsilon$ denote a positive number such that if each of $I$ and $I^{\prime}$ is an interval, and $f$ maps $I$ onto $I^{\prime}$ efficiently, and the length of $I^{\prime}$ is less than $\epsilon$, then $f$ is monotone on $I$. Let $K$ denote the subset of $[0,1]$ to which a number $x$ belongs if and only if there is a point $\left\{p_{i}\right\}$ in $\lim f$ such that $p_{1}=x$ and such that either (1) for some $i>0, p_{i} \in M$ or (2) for some $i>0, f^{-1}\left(p_{i}\right)$ contains an interval. $K$ is countable and so there is a finite collection $G^{\prime}$ of nonoverlapping intervals filling up $[0,1]$ such that each interval in $G^{\prime}$ is of length less than $\epsilon / 2$, and no endpoint of an interval in $G^{\prime}$, other than 0 or 1 , is a number in $K$. Let $G$ denote the intervals in $G^{\prime}$ which have neither 0 nor 1 as an endpoint. We now state an easily established lemma.

Lemma 1. If $g$ maps $[0,1]$ onto $[0,1]$ and $\alpha=\left\{\alpha_{i}\right\}$ is an inverse sequence for $g$, and $I$ is a subinterval of a term $\alpha_{n}$ of $\alpha$, then there is a subinverse sequence $\left\{\beta_{i}\right\}$ of $\alpha$ such that $\beta_{n}=I$ and for each $i>n, g$ maps $\beta_{i+1}$ onto $\beta_{i}$ efficiently.

Applying Lemma 1 , there is a subinverse sequence $A^{0}=\left\{A^{0}(i)\right\}$ of $A$ and an integer $n_{0}$ such that for each $i \geqq n_{0}, f$ maps $A^{0}(i+1)$ efficiently onto $A^{0}(i)$. If there is an $n$ such that for $i>n, f$ is monotone on $A^{0}(i)$, then $\lim \left(f, A^{0}\right)$ is an arc (see [1]). Since $\lim \left(f, A^{0}\right)$ is not an arc, there is a subsequence of $A^{0}$ each term of which is of length at least $\epsilon$ and so there is an interval $g_{1}$ in $G$ which is a subset of each term of some subsequence of $A^{0}$. Applying Lemma 1 again, there is a subinverse sequence $A^{1}=\left\{A^{1}(i)\right\}$ of $A^{0}$ and an integer $n_{1}$ such that $A^{1}\left(n_{1}\right)=g_{1}$ and such that if $i \geqq n_{1}, f$ maps $A^{1}(i+1)$ efficiently onto $A^{1}(i)$. As before we note that since $\lim \left(f, A^{1}\right)$ is not an arc, there is an interval $g_{2}$ in $G$ which is a subset of each term of some subsequence of $A^{1}$. We continue to establish the existence of a sequence $A^{0}, A^{1}$, $A^{2}, \cdots$, an increasing integer sequence $n_{1}, n_{2}, \cdots$ and a sequence $g_{1}, g_{2}, \ldots$ of members of $G$ such that for each $i>0$, (1) $A^{i}$ is a subinverse sequence of $A^{i-1}$, (2) $A^{i}\left(n_{i}\right)=g_{i}$, (3) if $j \geqq n_{i}, f$ maps $A^{i}(j+1)$ efficiently onto $A^{i}(j)$ and (4) $g_{i}$ is a subset of each term of a subsequence of $A^{i-1}$. There is an integer $u$ and an integer $m<u$ such that $g_{u}=g_{m}$. The sequence $A^{m}$ is a subinverse sequence of $A^{0}, A^{m}\left(n_{m}\right)=g_{m}$, and $g_{m}$ is a subset of each term of a subsequence of $A^{m}$. Further, as a consequence of the definition of $G$ we have that if $j \geqq n_{m}, f$ is not constant on any interval which contains an endpoint of $A^{m}(j+1)$ and it follows that $A^{m}(j+1)$ is a component of $f^{-1}\left[A^{m}(j)\right]$. Let $N$ denote an integer such that $A^{m}\left(n_{m}+N\right)$ contains $A^{m}\left(n_{m}\right)$. We shall show that if $j>0, A^{m}\left(n_{m}+N+j\right)$ contains $A^{m}\left(n_{m}+j\right)$. Either $A^{m}\left(n_{m}+N+1\right)$ 
$\supseteq A^{m}\left(n_{m}+1\right)$ or they do not intersect since $A^{m}\left(n_{m}+1\right)$ is a connected subset of $f^{-1}\left[A^{m}\left(n_{m}+N\right)\right]$ and $A^{m}\left(n_{m}+N+1\right)$ is a component of $f^{-1}\left[A^{m}\left(n_{m}+N\right)\right]$. Let $k$ denote a positive integer such that $A^{m}\left(n_{m}+N+k\right)$ contains $g_{m}$. Since $f^{N}\left(g_{m}\right) \subseteq g_{m}$, then $f^{N}\left[A^{m}\left(n_{m}+N+k\right)\right]$ intersects $g_{m}$ and thus intersects $A^{m}\left(n_{m}+N+k\right)$. But

$$
f^{N}\left[A^{m}\left(n_{m}+N+k\right)\right]=A^{m}\left(n_{m}+k\right),
$$

so $A^{m}\left(n_{m}+N+k\right)$ intersects $A^{m}\left(n_{m}+k\right)$ which implies that $A^{m}\left(n_{m}+N+1\right)$ intersects, and thus contains, $A^{m}\left(n_{m}+1\right)$. It follows inductively that if $j>0, A^{m}\left(n_{m}+N+j\right)$ contains $A^{m}\left(n_{m}+j\right)$. Now let $g=f^{N}$ and for each $j>0$ let $B(j)=A^{m}\left[n_{m}+(j-1) N\right]$. Then $g$ is a piecewise monotone map of $[0,1]$ onto $[0,1], B=\{B(i)\}$ is an inverse sequence (for $g$ ), and for each $j>0, B(j+1) \supseteq B(j)$ and $g$ maps $B(j+1)$ efficiently onto $B(j)$. Further, $\lim (g, B)$ is homeomorphic to a subcontinuum of $\lim (f, A)$ and so the following lemma applies to complete our proof.

LEMMA 2. If $g$ maps $[0,1]$ onto $[0,1]$ and is piecewise monotone and $B=\left\{B_{i}\right\}$ is an inverse sequence (for $g$ ) such that for each $i>0, B_{i}$ is a subinterval of $B_{i+1}$ and $g$ maps $B_{i+1}$ efficiently onto $B_{i}$, then $\lim (g, B)$ contains an arc.

Proof. For each $i>0$, let $B_{i}=\left[a_{i}, b_{i}\right]$. The sequence $\left\{a_{i}\right\}$ is nonincreasing and converges to a number $a$. Similarly, $\left\{b_{i}\right\}$ converges to a number $b$. Further, there is a number $x$ such that the point $(x, x, \cdots)$ is in $\lim (g, B)$. Suppose that there is a positive integer $n$ such that $g\left(b_{n+2}\right)=a_{n+1}$ and $g\left(a_{n+1}\right)=a_{n}$. According as $g\left(b_{n+3}\right)=b_{n+2}$ or $g\left(b_{n+3}\right)$ $=a_{n+2}$, we have that either $g\left(\left[b_{n+2}, b_{n+3}\right]\right)$ contains $x$ and is nondegenerate or $g\left(\left[a_{n+3}, a_{n+1}\right]\right)$ contains $x$ and is nondegenerate. But $g$ is piecewise monotone and there do not exist infinitely many mutually disjoint intervals such that if $I$ is one of them, $g(I)$ contains $x$ and is nondegenerate. So there is an integer $N^{\prime}$ such that if $n>N^{\prime}$, then it is not true that $g\left(b_{n+2}\right)=a_{n+1}$ and $g\left(a_{n+1}\right)=a_{n}$. It follows that there is an integer $N$ such that either (1) if $n>N, g\left(a_{n+1}\right)=a_{n}$, or (2) if $n>N$, $g\left(a_{n+1}\right)=b_{n}$. Suppose first that (1) holds and that $b \neq x$. There is a number $u, x<u<b$, such that $g$ is monotone on $[u, b]$ and an integer $k$ such that if $j \geqq k, b_{j}>u$. If $g(u) \leqq u$, there is a point $\left\{u_{i}\right\}$ in $\lim g$ such that $u_{1}=u$ and for $i>0, u_{i} \leqq u_{i+1}<b_{k+i}$. Then for $i>0$, $g\left(\left[u_{i+1}, b_{k+i}\right]\right)=\left[u_{i}, b_{k+i-1}\right]$ and $g$ is monotonic on $\left[u_{i+1}, b_{k+i}\right]$. It follows that the set of all points $\left\{p_{i}\right\}$ of $\lim g$ such that for $i>0, p_{k+i}$ $\in\left[u_{i+1}, b_{k+i}\right]$ is an arc which is a subset of $\lim (g, B)$. If $g(u)>u$, then there is a number $s$ and a number $t$ such that (1) $g(s)=s$, (2) $x \leqq s$ $<t<u$, (3) if $y \in[s, t], g(y) \geqq y$ and (4) $g$ is monotonic on $[s, t]$. There 
is a point $\left\{t_{i}\right\}$ in $\lim g$ such that $t_{1}=t$ and for each $i>0, s<t_{i+1} \leqq t_{i}$. For each $i>0, g\left(\left[s, t_{i+1}\right]\right)=\left[s, t_{i}\right]$ and $g$ is monotonic on $\left[s, t_{i+1}\right]$, and it follows that the set of all points $\left\{p_{i}\right\}$ of $\lim g$ such that for $i \geqq 0$, $p_{k+i} \in\left[s, t_{i+1}\right] \subseteq\left[a_{k+i}, b_{k+i}\right]$ is an arc which is a subset of $\lim (g, B)$. Thus we have that $\lim (g, B)$ contains an arc in case there is an integer $N$ such that if $n>N, g\left(a_{n+1}\right)=a_{n}$ and $b \neq x$. An analogous argument suffices in case $a \neq x$ and it remains to show that $\lim (g, B)$ contains an arc in case there is an integer $N$ such that if $n>N, g\left(a_{n+1}\right)=b_{n}$. In this case we consider the piecewise monotone map $g^{2}$ of $[0,1]$ onto $[0,1]$ and the inverse sequence $B^{\prime}=B_{1}, B_{3}, B_{5}, \cdots$. There is an integer $k$ such that if $i>k, g^{2}\left(a_{2 i+1}\right)=a_{2 i-1}$, so an argument analogous to that given above applies to show that $\lim \left(g^{2}, B^{\prime}\right)$ contains an arc. But $\lim (g, B)$ is homeomorphic to $\lim \left(g^{2}, B^{\prime}\right)$ and this completes our proof.

\section{REFERENCES}

1. C. E. Capel, Inverse limit spaces, Duke Math. J. 21 (1954), 233-245.

2. H. Freudenthal, Entwicklungen von Räumen und ihren Gruppen, Compositio Math. 4 (1937), 145-234.

3. G. W. Henderson, The pseudo-arc as an inverse limit with one binding map, Duke Math. J. 31 (1964), 421-425.

4. W. S. Mahavier, A chainable continuum not homeomorphic to an inverse limit on $[0,1]$ with only one bonding map, Proc. Amer. Math. Soc. 18 (1967), 284-286.

5. S. Mardesić and J. Segal, $\epsilon-m a p p i n g s$ and inverse limits, Trans. Amer. Math. Soc. 109 (1963), 146-164.

EMORY UNIVERSITY 